\title{
HERPES ZOSTER CHORIORETINOPATHY
}

\author{
TIMOTHY V. ROBERTS ${ }^{1}$, IAN C. FRANCIS ${ }^{2}$, MEDDUMA B. KAPPAGODA ${ }^{2}$ and \\ ANDREW D. DICK ${ }^{3}$ \\ Sydney, Australia
}

\begin{abstract}
SUMMARY
Chorioretinitis and subsequent choroidal and retinal pigment epithelial atrophy following herpes zoster ophthalmicus (HZO) have rarely been reported. We report two patients, who several months following attacks of acute HZO, developed posterior fundus features of yellow, non-pigmented, punched-out areas of retinal pigment epithelial and choroidal pigment atrophy, which we have termed herpes zoster chorioretinopathy. An occlusive vasculitic process is proposed as the pathogenesis for this chorioretinopathy, and may be similar to that seen in the delayed cerebral vasculitis following HZO. A previous history of HZO should be sought in patients with a unilateral, multifocal, nonpigmented chorioretinopathy, as this may represent a characteristic delayed feature.
\end{abstract}

The ophthalmic and systemic manifestations of herpes zoster infection are protean and have been well described. ${ }^{1-5}$ In herpes zoster ophthalmicus (HZO), involvement of the posterior segment of the eye is relatively uncommon. ${ }^{1}$ Delayed contralateral hemiparesis due to a cerebral vasculitis is now a well-documented syndrome, ${ }^{5-8}$ the pathogenesis of which has been confirmed by angiographic and histopathological evidence of vasculitis of large and small cerebral arteries. ${ }^{5,9}$ Similarly, histopathological studies of enucleated eyes following HZO show granulomatous arteritis involving the posterior ciliary arteries. ${ }^{2,3} \mathrm{We}$ report two cases of chorioretinopathy with areas of yellow, non-pigmented, punched-out atrophy of the retinal pigment epithelium and choroid. Their delayed onset is similar to that seen with cerebral vasculitis following HZO. The exact pathogenesis of the chorioretinal lesions described in our two patients is unclear, but the possibilities are occlusive vasculitis, direct lytic viral infection, or a combination of both. ${ }^{5}$

From: ${ }^{1}$ Westmead Hospital, ${ }^{2}$ Concord Hospital and ${ }^{3}$ Centenary Institute of Cell Biology and Cancer Medicine, Sydney, Australia.

Correspondence to: Dr Ian C. Francis, Suite 1, Malvern Court, 16-18 Malvern Avenue, Chatswood NSW 2067, Australia.

\section{Case 1}

CASE REPORTS

A 67-year-old man presented in September 1987 with decreasing vision in the left eye, 2 weeks following an attack of left-sided HZO. Visual acuity was counting fingers only. The cornea and bulbar conjunctiva were anaesthetic, and a large neurotrophic central corneal ulcer was present. There was mild anterior chamber activity. The left pupil was slightly larger than the right and reacted sluggishly to light and demonstrated light-near dissociation. The retina was normal, but there were a few cells in the anterior vitreous.

Treatment was commenced with guttae atropine, chloramphenicol and dexamethasone, and the eye was double-padded. The patient's visual symptoms improved, and 8 weeks later the corneal ulcer had healed, the anterior chamber was quiet, and the retina was still normal in appearance. Visual acuity improved to $6 / 5$.

Eight months later, in July 1988, he presented again with decreased vision in the left eye. Visual acuity was $6 / 12$, there was mild anterior chamber activity, a few cells in the vitreous, and a relative afferent pupil defect, grade $1 / 4$. The optic disc was slightly swollen, and areas of chorioretinal atrophy were seen (Fig. 1). A diagnosis of herpes zoster chorioretinopathy (HZCR) and vasculitic ischaemic optic neuropathy was made.

Fluorescein angiography showed areas of window defects corresponding to the areas of chorioretinopathy (Fig. 2), and leakage at the optic disc. Full blood count, biochemistry and erythrocyte sedimentation rate were normal, and his HIV status was negative.

In view of the posterior segment involvement, consideration was given to commencing treatment with systemic acyclovir and prednisone. However, the episode resolved spontaneously, and by November 1988 the vision was back to 6/5. The optic disc appeared healthy, and the areas of chorioretinal atrophy unchanged. At the last examination in 


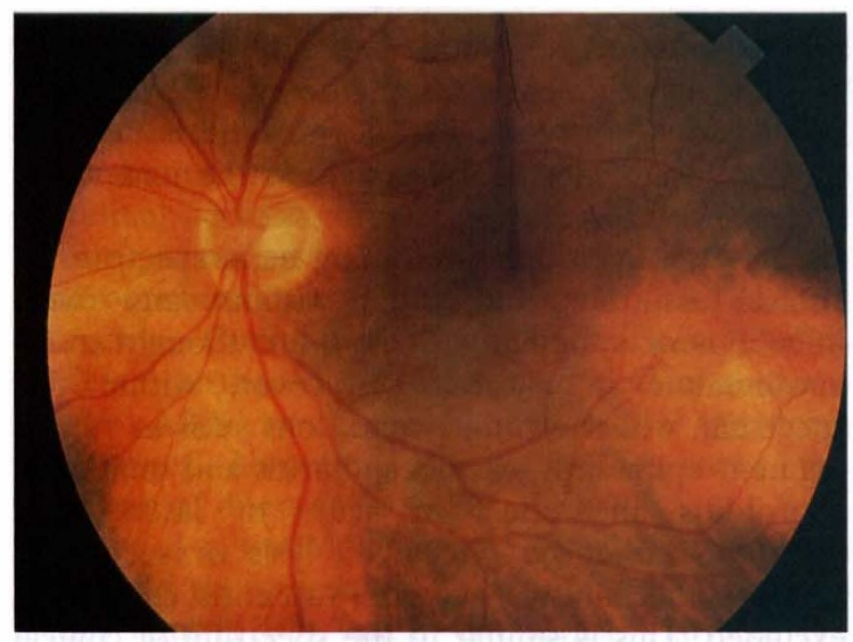

Fig. 1. Case 1. Left eye. Multifocal chorioretinal atrophy 8 months after initial presentation with $\mathrm{HZO}$.

November 1993, more than 6 years after the initial episode the vision was still $6 / 6$, with an early cortical cataract forming, but there had been no change in the fundal appearance. The patient remained systemically well.

\section{Case 2}

A 73-year-old woman was first seen in November 1983, 9 days after the onset of right-sided acute HZO. Visual acuity was $6 / 9$ part. There was a mild anterior uveitis, and diffuse punctate epithelial keratopathy. Treatment was commenced with topical chloramphenicol, dexamethasone and homatropine. Immediately following the acute attack, she developed severe post-herpetic neuralgia requiring oral prednisone. The fundus was normal at that time.

Five months later, in March 1984, she presented with an inflamed right eye, anterior uveitis and posterior synechiae. The cornea was clear and fundal examination revealed depigmented lesions at the

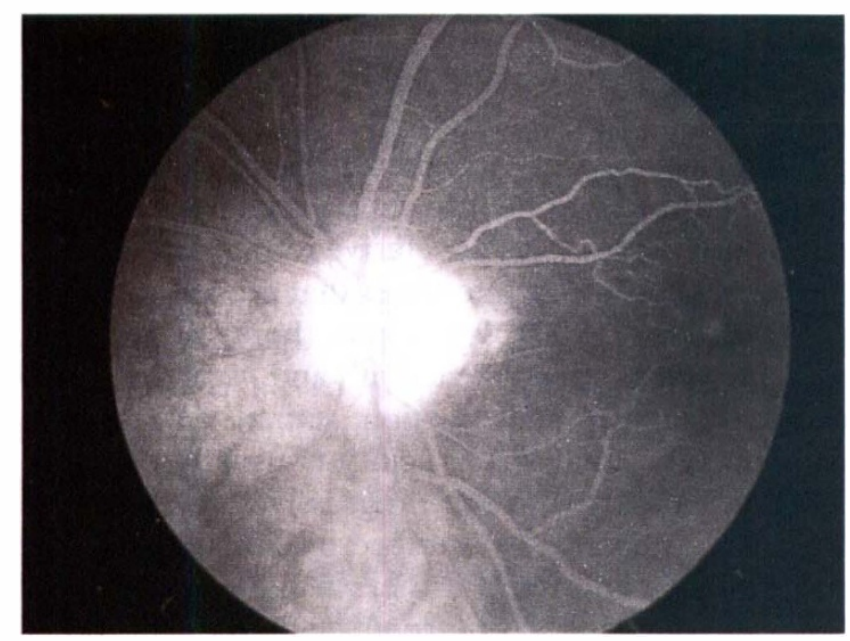

Fig. 2. Case 1. Corresponding fluorescein angiogram to Fig. 1, showing window defects, with increased transmission of choroidal fluorescence, and leakage at the optic disc.

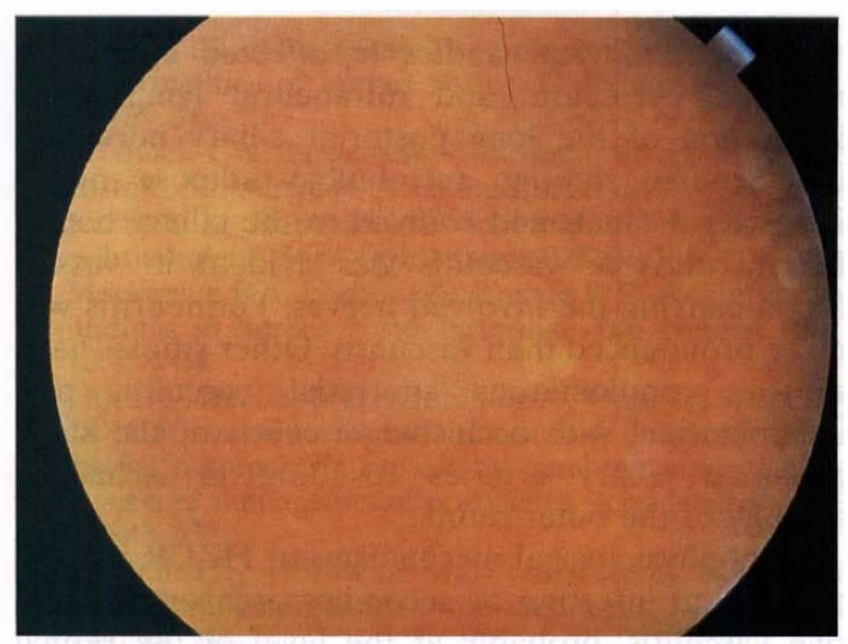

Fig. 3. Case 2. Right eye. Scattered multifocal areas of chorioretinal atrophy 4 months after initial attack of $\mathrm{HZO}$ (view obscured by cataract).

level of the choriocapillaris/retinal pigment epithelium in the superior macular region, extending to the superotemporal arcade.

When reviewed 2 months later, more retinal lesions had developed in the perifoveal region extending to the nasal fundus, but the visual acuity was still 6/6. By 1987, a posterior subcapsular cataract had developed, but the retinal lesions were unchanged and appeared identical to those seen in case 1 (Fig. 3). The iris showed sectoral atrophy with a poorly reactive pupil and light-near dissociation.

When last seen in 1994, the patient's visual acuity was perception of light, because of a dense, nuclear sclerotic white cataract. The cornea remained clear. The patient-declined cataract surgery at this stage.

\section{DISCUSSION}

Both in our experience, and in review of the literature, chorioretinal atrophy following HZO is a rare complication. ${ }^{1,3-5}$ Womack and Liesegang ${ }^{4}$ reviewed 86 patients seen with $\mathrm{HZO}$, and found none with optic nerve or retinal involvement. Four of the 86 had neurological complications, including 2 patients with contralateral hemiplegia, and 2 with angiographically proven segmental cerebral angiitis. In the $1356 \mathrm{HZO}$ patients described by Marsh and Cooper, ${ }^{1}$ one case of 'retinal pigment epithelial degeneration' was mentioned. This patient apparently retained good vision. The authors described 'scarring around the macula' and it is therefore not certain whether their single case represents a further example of HZCR, but there is no mention of pigmentary changes in the fundus.

The possibility of HZCR is not mentioned in the review by Liesegang ${ }^{10}$ but a single case of central retinal artery occlusion and stroke following $\mathrm{HZO}$ was described in $1990,{ }^{11}$ and was felt by the authors to be the fourth reported case of central retinal artery occlusion complicating HZO. 
Histopathological studies of affected eyes have revealed perineural and intraneural lymphocytic infiltration of the long posterior ciliary nerves in their course through retrobulbar adipose tissue, intrascleral canals and choroid to the ciliary body. ${ }^{2}$ Perivasculitis or vasculitis was evident in vessels accompanying the involved nerves. Perineuritis was more pronounced than vasculitis. Other studies have shown granulomatous choroidal vasculitis and inflammation, with occlusive vasculitis of the short posterior ciliary arteries resulting in ischaemic atrophy of the outer retina. ${ }^{3}$

Pathophysiological mechanisms of HZCR include direct viral infection or secondary ischaemic occlusive vasculitis, probably at the level of the retinal pigment epithelium (RPE) or choriocapillaris respectively, in patients with HZO. RPE and choroidal vascular endothelium express adhesion molecules to which most viruses will adhere and induce a direct lytic response or indirect CD8+ T-cell lysis. ${ }^{12}$ Despite electron microscopy showing evidence of virus particles in sections of iris tissue and retina of patients with $\mathrm{HZO},{ }^{13,14}$ the mechanism of HZCR is probably more likely to be an occlusive vasculitis resulting from, for example, immune complex deposition in the wall of the affected vessel, with subsequent binding of complement and the attraction of acute inflammatory cells to the area. ${ }^{15}$ The latency of presentation in HZCR contrasts with the acute retinal necrosis-type syndromes, and the lack of associated severe vitritis and retinitis in HZCR compared with acute retinal necrosis (ARN). Varicella zoster virus retinal infection has been shown to be a cause of retinal necrosis, ${ }^{16}$ but the clinical picture is characteristically different from that of $\mathrm{HZO}$.

As we have mentioned, contralateral hemiplegia complicating $\mathrm{HZO}$ is a well-defined clinical syndrome whose pathogenesis appears to parallel that of HZCR. The interval between the onset of zoster and cerebral vasculopathy ranges from 0 to 6 months. ${ }^{7}$ Angiography reveals segmental constriction of the major intracerebral arteries or abnormalities of the carotid syphon. ${ }^{6}$ Cases have been reported, though, of patients with normal carotid angiography but occlusive granulomatous angiitis of small vessels. ${ }^{8}$ One report of a patient with $\mathrm{HZO}$ and central nervous system involvement found granulomatous angiitis involving the basilar artery, and electron microscopic evidence of virus-like particles in the outer layers of the vessel walls, ${ }^{9}$ suggesting direct viral invasion of blood vessels and resultant granulomatous angiitis. It does appear, therefore, that HZO may be complicated by arteritis affecting vessels of any size in the cerebral or ocular circulation.

The similar temporal delay in development of
HZCR and hemiplegia, and the histopathological findings of granulomatous angiitis in both cerebral and choroidal vessels, ${ }^{2,4}$ may support the assumption that occlusive choroidal vasculitis is the underlying mechanism in HZCR.

Posterior uveitides, presenting as a spectrum of clinical disorders and often without any overt infectious or systemic aetiology, are thought to be autoimmune in nature. ${ }^{12}$ However, within this spectrum, which includes conditions such as retinal pigment epitheliitis, choriocapillaritis and multifocal choroiditis, the effect of acute or latent viral infections either by direct cell lysis or secondary immune mechanisms such as vasculitis cannot be excluded. This is similar to the spectrum of clinical signs seen in post-viral (including zoster) uveitis. ${ }^{17}$

A spectrum of chorioretinal changes associated with herpes zoster infection has been demonstrated by three reports which described atypical fundus features associated with $\mathrm{HZO}$, and similar to our two cases of HZCR. Amano and associates ${ }^{18}$ described three patients with many punched-out oval areas of choroidal atrophy scattered around the mid-periphery but without associated pigmentary changes. Bloom and Snady-McCoy ${ }^{19}$ described a 67-year-old man with 'multifocal choroiditis uveitis' occurring after HZO. McElvanney and Murray ${ }^{20}$ more recently described a patient with the delayed development of multifocal choroidal lesions.

The evidence that viral infections may induce similar clinical appearances has been described in acute systemic chicken pox infection which produces an inflammatory chorioretinitis, ${ }^{21,22}$ the late fundal appearance of which resembles that seen in our two patients with HZCR. In these cases, however, an immediate and direct viral aetiology seems more likely since the single patient described in each of these case reports had acute chicken pox at the time of developing the chorioretinitis.

By contrast, at the other end of the spectrum, it appears that the acute retinal necrosis (ARN) syndrome presents a different clinical picture and a pathological process in that patients are usually young and in good health, and it is characterised by panuveitis, vitritis, vaso-occlusive retinal arteritis and necrotising retinitis ${ }^{23}$ - features that are absent in our cases. In the ARN syndrome there is generally marked pigmentary reaction and scarring, and rhegmatogenous and traction retinal detachments develop. The final visual acuity is usually poor. ${ }^{23,24}$ Even in patients with the Progressive Outer Retinal Necrosis syndrome, ${ }^{25}$ described by Forster et al., the visual acuity suffered. Further, in the so-called mild type of the ARN syndrome ${ }^{26}$ there are retinal exudates and arteritis in the setting of acute systemic illness. In our patients with HZCR, the acute illness had resolved several months previously. 
While vision may not suffer in the mild cases, this is the exception, not the rule, and in our cases of HZCR, and in those in the literature ${ }^{18-20}$ the final visual acuity remained intact.

Retinal necrosis, of varying severity, appears still to be a prominent feature of the disease in the ARN syndrome but not in HZCR. In fact, Holland and the Executive Committee of the American Uveitis Society ${ }^{27}$ further emphasised the aspect of necrosis in the ARN syndrome by suggesting the term 'necrotising herpetic retinopathy' to cover the spectrum of disease.

There is a well-documented association between $\mathrm{HZO}$ and the presence of the human immunodeficiency virus (HIV), and in one series, ${ }^{28} 26 \%$ of 112 patients with HZO had HIV present. ARN developed in 5 of the 112 patients, but typically after the full-blown syndrome developed. It was also bilateral in 4 of the 5 patients. It was an immediate, and not a delayed, phenomenon.

Of note in our cases of HZCR and the others described in the literature $e^{18-20}$ is the complete absence of pigmentary reaction associated with zoster chorioretinal scarring. Presumably the absence of a pigmentary reaction is due to destruction of the reactive RPE in the affected area. This contrasts with most infectious vascular and inflammatory insults to the choriocapillaris/RPE, which result in pigmented scarring.

HZCR is uncommon and, in fact, Professor J. D. M. Gass, MD (personal communication, August 1994), who co-authored one of the original ocular pathological studies of $\mathrm{HZO}^{2}$, said that he may possibly have seen just one case about 25 years previously. Similarly, Mr R. J. Marsh, FRCS (personal communication, January 1994), emphasised again the rarity of the disorder; he had in fact only one possible case to report. ${ }^{1}$

HZCR also appears to have several distinct features. These include the delayed presentation after HZO; the presence of single or multifocal nonpigmented lesions with no foveal involvement or retinal detachment; the self-limiting and benign nature; and the selective nature of the depigmentation without associated pigment scarring and clumping.

We feel that in patients with a unilateral, nonpigmented chorioretinopathy, a previous history of HZO should be sought, as this may represent a characteristic delayed feature of this disease.

Key words: Delayed chorioretinopathy, Herpes zoster.

\section{REFERENCES}

1. Marsh RJ, Cooper M. Ophthalmic herpes zoster. Eye 1993;7:350-70.

2. Naumann G, Gass JDM, Font RL. Histopathology of herpes zoster ophthalmicus. Am J Ophthalmol 1968; 65:533-41.
3. Hedges TR, Albert DM. The progression of the ocular abnormalities of herpes zoster. Ophthalmology 1982; 89:165-76.

4. Womack LW, Liesegang TJ. Complications of herpes zoster ophthalmicus. Arch Ophthalmol 1983;101:42-5.

5. Karbassi M, Raizman MB, Schuman JS. Herpes zoster ophthalmicus. Surv Ophthalmol 1992;36:395-410.

6. Mackenzie RA, Forbes GS, Karnes WE. Angiographic findings in herpes zoster arteritis. Ann Neurol 1981; 10:458-64.

7. Hilt DC, Buchholz D, Krumholz A, Weiss H, Wolinsky JS. Herpes zoster ophthalmicus and delayed contralateral hemiparesis caused by cerebral angiitis: diagnosis and management approaches. Ann Neurol 1983; 14:543-53.

8. McNeil JD, Horowitz M. Contralateral hemiplegia complicating herpes zoster ophthalmicus. J R Soc Med 1991;84:501-2.

9. Linnemann CC, Mariano MM. Pathogenesis of varicella-zoster angiitis in the CNS. Arch Neurol 1980;37:239-40.

10. Liesegang TJ. Diagnosis and therapy of herpes zoster ophthalmicus. Ophthalmology 1991;98:1216-29.

11. Wilson CA, Wander AH, Choromokos EA. Central retinal artery obstruction in herpes zoster ophthalmicus and cerebral vasculopathy. Ann Ophthalmol 1990;22: 347-51.

12. Forrester JV. Uveitis: pathogenesis. Lancet 1992;338: 1489-90.

13. Witmer R, Iwamoto T. Electron microscopy observation of herpes-like particles in the iris. Arch Ophthalmol 1968;79:331-7.

14. Schwartz JN, Cashwell F, Hawkins HK, Klintworth GK. Necrotizing retinopathy with herpes zoster ophthalmicus. Arch Pathol Lab Med 1976;100:386-91.

15. O'Connor GR. Herpes zoster uveitis. In: GrausMackiw E, O'Connor GR (eds). Uveitis: pathophysiology and therapy. New York: Thieme-Stratton, 1983:55-6.

16. Culbertson WW, Blumenkranz MS, Pepose JS, Stewart JA, Curtin VT. Varicella zoster virus is a cause of the acute retinal necrosis syndrome. Ophthalmology 1986;93:559-69.

17. Dua HS, Dick AD, Watson NJ, Forrester JV. A spectrum of clinical signs in anterior uveitis. Eye 1993;7:68-73.

18. Amano Y, Ohashi Y, Haruta Y, Kinoshita S, Tano Y, Manabe R. A new fundus finding in patients with zoster ophthalmicus. Am J Ophthalmol 1986;102: 532-3.

19. Bloom SM, Snady-McCoy L. Multifocal choroiditis uveitis occurring after herpes zoster ophthalmicus. Am J Ophthalmol 1989;108:733-5.

20. McElvanney AM, Murray PI. Multifocal choroidal lesions: a rare complication of herpes zoster chorioretinopathy. J Neuro-Ophthalmol 1994;14:12-4.

21. Kelly SP, Rosenthal AR. Chicken pox chorioretinitis. Br J Ophthalmol 1990;74:698-9.

22. Deegan W. F., Duker JS. Unifocal choroiditis in primary varicella zoster (chicken pox). Arch Ophthalmol 1994;112:735-6.

23. Gartry DS, Spalton DJ, Tilzey A, Hykin PG. Acute retinal necrosis syndrome. Br J Ophthalmol 1991; 75:292-7.

24. Matsuo T, Morimoto K, Matsuo N. Factors associated with poor visual outcome in acute retinal necrosis. Br J Ophthalmol 1991;75:450-4.

25. Forster DJ, Dugel PU, Frangieh GT, Liggett PE, Rao NA. Rapidly progressive outer retinal necrosis in the 
acquired immunodeficiency syndrome. Am J Ophthalmol 1990;110:341-8.

26. Matsuo T, Makauama T, Koyama T, Koyama M, Matsuo N. A proposed mild type of the acute retinal necrosis syndrome. Am J Ophthalmol 1988;105:579-83.

27. Holland GN, and the Executive Committee of the American Uveitis Society. Standard diagnostic criteria for the acute retinal necrosis syndrome. Am J Ophthalmol 1994;117:663-6.

28. Sellitti TP, Huang AJW, Schiffmann MS, Davis JL. Association of herpes zoster ophthalmicus with acquired immunodeficiency syndrome and acute retinal necrosis. Am J Ophthalmol 1993;116:27-30. 